\title{
Di controlleria sui treni delle Ferrovie dello Stato Italiane non si parla più (ma il controllo dei biglietti resta!)
}

Paolo D'Achille

PUBBLICATO: 04 GIUGNO 2019

\section{Quesito:}

Nel corso del 2017 sono arrivate in Accademia varie segnalazioni di un annuncio ferroviario (diffuso in particolare sui treni regionali) che invitava i viaggiatori a tenere pronti il biglietti (anzi, i "titoli di viaggio") perché di li a poco sarebbe iniziata "l'attività di controlleria". Molti esprimevano fastidio per l'uso di questa parola da parte delle Ferrovie dello Stato Italiane, anche se qualcuno ammetteva di averla trovata registrata in qualche dizionario. Ma da qualche tempo l'annuncio è cambiato...

D i controlleria sui treni delle Ferrovie dello Stato Italiane non si parla più (ma il controllo dei biglietti resta!)

U na breve premessa, per rispondere ad altre domande che ci sono pervenute e che riguardano la denominazione delle Ferrovie. Il nome ufficiale dell'azienda è oggi Ferrovie dello Stato Italiane (e non Ferrovie dello Stato Italiano) e l'accordo dell'aggettivo con la testa della locuzione, pienamente corretto, vuole probabilmente sottolineare l'italianità delle linee ferroviarie. La sigla attuale è FS; FFSS (o anche FF.SS.) si usava in passato e la ripetizione delle consonanti si giustificava col fatto che nelle abbreviazioni dei plurali in italiano si raddoppiano spesso le consonanti: si pensi a pp. o pagg. (pagine) opposto a p. o pag. (pagina), a ss. o sgg. (seguenti) rispetto a $s$. o sg. (seguente), a proff. (professori) rispetto a prof. (professore). Ma poi ha, evidentemente, prevalso l'dea di semplificare la sigla, anche perché la sua pronuncia più frequente (effe-effe-esse-esse) aveva un che di buffo o comunque la rendeva poco perspicua.

E veniamo ora al quesito. La parola controlleria nel senso di 'controllo del possesso e della validità dei biglietti' esiste in italiano almeno dal sec. XVIII: è registrata infatti nel GRADIT con la data I769 (che si riferisce all'attestazione in Pietro Verri riportata nel GDLI) e con esempi come personale di c(ontrolleria), addetti alla c(controlleria).

Analogamente a quanto segnalato su questa stessa rubrica da Rita Librandi a proposito del termine bidelleria, che è addirittura più antico ma che è stato accolto nei dizionari solo di recente, si tratta di una parola ben formata. Come mai, allora, il suo uso ha suscitato tante perplessità? Forse perché controlleria in questo caso non indica il luogo in cui si effettuano i controlli ma l'attività di controllo? Ora, è vero che il suffisso -eria è produttivo per formare, a partire da nomi, nomi collettivi e, soprattutto, nomi di luogo, ma può essere usato anche per formare nomi che indicano attività e azione (cfr. i vari saggi che ne trattano nel fondamentale volume La formazione delle parole in italiano, a cura di Maria Grossmann e Franz Rainer, Tübingen, Niemeyer, 2004).

Una spiegazione alternativa ce la fornisce lo stesso GRADIT, che accoglie si la parola nel suo lemmario, ma segnala che è di B(asso) U(so). Si tratta infatti di un termine rimasto sempre confinato nell'àmbito burocratico, e si sa che la burocrazia predilige forme desuete o rare, a cui conferisce spesso lo statuto di tecnicismi. Ma la collocazione di controlleria allinterno di un annuncio destinato a viaggiatori, la maggior parte dei quali non aveva mai incontrato prima d'allora quel termine, tanto da 
sentirlo come un neologismo e da rimanere perplessa o addirittura infastidita dal suo uso, è sembrata poco opportuna.

Cosi, quando, nello stesso 2017, le Ferrovie hanno chiesto la collaborazione dell'Accademia della Crusca per rivedere gli annunci, tra le proposte che abbiamo avanzato (e che le Ferrovie hanno accolto), accanto alla sostituzione di "clienti" con "viaggiatori" e di "esibire il titolo di viaggio" con "mostrare il biglietto", all'invito a pronunciare "dispórsi" (con la o chiusa) e non "dispórsi" (con la o aperta), c'è stato anche il cambiamento del nostro annuncio: al posto dell'" attività di controlleria" ci sono ora le "operazioni di controllo". Di controlleria, dunque, in treno ormai non si parla più; resta però il controllore, che continua, giustamente, a verificare il possesso del biglietto da parte dei singoli viaggiatori.

\section{Cita come:}

Paolo D'Achille, Dicontrolleria sui treni delle Ferrovie dello Stato Italiane non si parla più (ma il controllo dei biglietti resta!), "Italiano digitale", 2019, IX, 2019/2 (aprile-giugno)

DOI: 10.35948/2532-9006/2020.3121

\section{Copyright 2019 Accademia della Crusca}

Pubblicato con licenza creative commons CC BY-NC-ND 